\title{
THERMAL RADIO EMISSION FROM NORMAL GALAXIES
}

\author{
YERVANT TERZIAN \\ Cornell University, Ithaca, N.Y., U.S.A.
}

\begin{abstract}
Thermal radiation from normal spiral galaxies may be detectable at centimeter and millimeter wavelengths. Predictions have been made assuming free-free radiation from $\mathrm{HII}$ regions at $T_{e}=7000 \mathrm{~K}$, and a range of mean electron densities and radii.
\end{abstract}

\section{Introduction}

The observed radio spectra of normal galaxies are characteristic of the type of spectra found for non-thermal sources. However, thermal emission due to free-free transitions in ionized hydrogen regions inside normal galaxies also contributes to the radio radiation. Observations and theory suggest that the non-thermal radiation from normal galaxies is more intense than the thermal radiation by several orders of magnitude at radio frequencies below $5 \mathrm{GHz}$. At higher frequencies the contribution of the thermal radiation becomes important and perhaps it may even be the dominant source of energy at frequencies higher than $30 \mathrm{GHz}$.

This paper is a preliminary study in estimating the contribution of the thermal radiation from normal galaxies. Recently Hodge (1969) has completed the work 'An Atlas and Catalog of Hir Regions in Galaxies'. This work is a search for $\mathrm{H} \alpha-$ emission in ninety galaxies which led to the discovery of $\mathrm{H}$ II regions in sixty of them. Here we use Hodge's work for nine galaxies to estimate their thermal radio emission from the catalogued Hil regions. In this study we also include the Andromeda galaxy (M31) using the HII data from Baade and Arp (1964), and M33 using the data by Sandage (1962), Courtès and Cruvellier (1965), and Carranza et al. (1968).

The predicted thermal radio spectra are then compared with the available radio observations. These observations are primarily from Mathewson and Rome (1963), Heeschen and Wade (1964), De Jong (1966, 1967), Lang and Terzian (1969), Kuril'chick et al. (1970), and Whiteoak (1970). The radio observations for M31 are from Kraus et al. (1966), and Howard and Maran (1965).

\section{Thermal Emission}

The available data on emission nebulae in normal galaxies put some restrictions on the methods one can use to compute the thermal radio emission. The most reliable technique for computing the thermal radio emission is to use the recombination theory together with observed absolute fluxes of Balmer lines integrated over an entire galaxy. Terzian $(1965,1968)$ has shown that the ratio of the fluxes at a radio frequency $v$ and at $\mathrm{H} \beta$ for an optically thin thermal region is given as

$$
S_{v}=\frac{N_{i}}{S_{p}} \frac{T_{e}}{N_{p}}\left\langle\frac{\left.1.664 \times 10^{-19}\right\rangle}{e^{0.986 \times 10^{4 / T} / T_{e}}} \ln \left(49.5 \frac{T_{e}^{3 / 2}}{v}\right)\right.
$$


where $N_{i} / N_{p}$ is the ratio of the number of ions to protons and indicates the relative abundance of helium, which can be estimated to be 10 to $15 \% . T_{e}$ is the electron temperature which is of the order of $10^{4} \mathrm{~K},\left\langle b_{4}\right\rangle$ describes the departure from thermodynamic equilibrium for the $\mathrm{H} \beta$ transition and it is equal to 0.20 (Burgess, 1958), and $v$ is the radio frequency. Clearly the dependence on frequency for an optically thin thermal source is very small. If we let $v=5 \mathrm{GHz}$, we find that

$$
\frac{S_{v}}{S_{H \beta}}=3.2 \times 10^{-14} .
$$

Hence the thermal radio spectrum of a source can be computed if one has a knowledge of the $\mathrm{H} \beta$ flux. Similarly a knowledge of the fluxes of other Balmer or Paschen lines may allow us to predict the thermal radio spectra (Pipher and Terzian, 1969).

Unfortunately the above method cannot be used for the present study because absolute Balmer line fluxes are not available for Hil regions in normal galaxies. One should emphasize that such data will be extremely useful in computing the free-free emission from other galaxies.

In this preliminary study we compute the thermal radio spectra making use of the free-free emission theory together with the total number (and apparent area) of the emission regions in each galaxy measured from the work of Hodge. Since the measurement of emission region areas is a very uncertain quantity, we also compute thermal spectra by assuming certain mean sizes to the emitting regions.

The flux density of a radio source is normally given as

$$
S_{v}=\frac{2 k v^{2}}{c^{2}} \int_{\Omega} T_{b} \mathrm{~d} \Omega
$$

where $k$ is Boltzmann's constant, $c$ is the velocity of light, $T_{b}$ is the brightness temperature, and $\Omega$ is the solid angle of the source. If one assumes constant brightness for a source, then letting $\Omega=A / D^{2}$ where $A$ is the projected area of the source and $D$ is its distance, we can then write

$$
S_{v}=\frac{2 k v^{2}}{c^{2}} \frac{T_{b} A}{D^{2}}
$$

The brightness temperature is obtained from the solution of the equation of radiative transfer as $T_{b}=T_{e}\left(1-e^{-\tau_{\nu}}\right)$, where $\tau_{v}$ is the optical depth. Since the thermal radio emission will only be important at the very high frequencies where generally $\tau_{v} \ll 1$, we can approximate $T_{b} \approx T_{e} \tau_{v}$, and the last expression becomes

$$
S_{v}=\frac{2 k v^{2}}{c^{2}} \frac{A T_{e} \tau_{v}}{D^{2}}
$$

The optical depth for thermal emission can be derived from the free-free absorption coefficient (Oster 1961, Terzian 1968) as

$$
\tau_{v}=\frac{9.78 \times 10^{-3}}{v^{2} T_{e}^{3 / 2}} \ln \left(4.95 \times 10^{7} \frac{T_{e}^{3 / 2}}{v}\right) \int N_{e}^{2} \mathrm{~d} s
$$


where $N_{e}$ is the electron density in $\mathrm{cm}^{-3}$ and $v$ is the frequency in units of $\mathrm{Hz}$. One can assume that the HII regions inside a galaxy are spherical and have uniform electron densities, so that $\int N_{e}^{2} \mathrm{~d} s=N_{e}^{2} s$ where $s$ is the diameter of a single emission region in a galaxy. If $A$ is the total projected area of emission regions in a galaxy and $N$ is the total number of such regions, then $A / N=\pi(s / 2)^{2}$, and $s=2(A / \pi N)^{1 / 2}$, so that

$$
N_{e}^{2} s=2 N_{e}^{2}\left(\frac{A}{\pi N}\right)^{1 / 2}
$$

Using Equations (6) and (7) with (5) we finally have

$$
S_{v}=\frac{3.91 \times 10^{-2} k}{c^{2}(\pi)^{1 / 2}} \frac{A^{3 / 2}}{D^{2}(N)^{1 / 2}} \frac{N_{e}^{2}}{\left(T_{e}\right)^{1 / 2}} \ln \left(4.95 \times 10^{7} \frac{T_{e}^{3 / 2}}{v}\right) .
$$

The last expression can now be used to compute the thermal radio spectra of normal galaxies. The distances for the galaxies are taken from Roberts (1969) and van den Bergh (1968). The values for $N$ are those given by Hodge (1969), Baade and Arp (1964) for M31, Sandage (1962) and Courtès and Cruvellier (1965) for M33.

TABLE I

Number of $\mathrm{HII}$ regions in galaxies and distances

\begin{tabular}{llll} 
Galaxy & Type & $\begin{array}{l}\text { Distance } \\
(\mathbf{M p c})\end{array}$ & $\begin{array}{l}\text { Number of } \\
\text { HII regions }\end{array}$ \\
\hline M31 & Sb & 0.69 & 688 \\
M33 & Sc & 0.69 & 369,101 \\
NGC 628 & Sc & 7.8 & 193 \\
NGC 2403 & Sc & 3.2 & 109 \\
NGC 2903 & Sc & 7.0 & 74 \\
NGC 3368 & Sa & 7.3 & 3 \\
NGC 3627 & Sb & 7.3 & 25 \\
NGC 3628 & Sb & 7.0 & 18 \\
NGC 4449 & IrI & 3.3 & 81 \\
NGC 5457 & Sc & 3.5 & 189 \\
NGC 6946 & Sc & 4.1 & 39 \\
\hline
\end{tabular}

Table I gives the distances to the galaxies and the number of $\mathrm{HII}$ regions in each of them. In the case of M33 Sandage has reported 369 emission nebulae and Courtès and Cruvellier 101. The latter work includes primarily the HII regions which were photographically resolved. In order to make use of Equation (8) one has to assume values for $T_{e}$ and $N_{e}$. From extensive research on emission nebulae in our own galaxy we find that $T_{e} \approx 7000 \mathrm{~K}$ with a variation of a few thousand degrees. Mean values for $N_{e}$ are more difficult to estimate; however, we know that in extreme cases $N_{e}$ may have values of $\sim 1 \mathrm{~cm}^{-3}$ to $\gtrsim 10^{6} \mathrm{~cm}^{-3}$ (as in compact $\mathrm{H}$ II regions). For the average regions, however, the range in $N_{e}$ can be taken to be $10 \mathrm{~cm}^{-3}$ to $100 \mathrm{~cm}^{-3}$.

In practice it is extremely difficult to measure reliable areas for most of the emission regions in other galaxies because of the limited resolution of the optical photographs. Nevertheless, we have tried to measure the total area of $\mathrm{H}_{\text {II }}$ regions given by Hodge 
for several galaxies and have applied the results in Equation (8). The results were very unrealistic. In particular the mean sizes of the emission regions were found to be several hundred parsecs in size as compared to 10-80 parsec for the sizes of normal $\mathrm{H}$ II regions in our own galaxy. However, one can argue that at distances of 3 to $7 \mathrm{Mpc}$ we detect only the giant $\mathrm{H}$ II regions.

For the reasons given above we have eliminated the parameter $A$ from Equation (8) and assumed several mean radii for the individual $\mathrm{H}$ II regions in other galaxies. Thus, thermal radio spectra were computed by using $A^{3 / 2}=r^{3}(\pi N)^{3 / 2}$ in Equation (8), where $r$ is the mean radius of an $\mathrm{H}$ II region.

\section{Results}

A series of computations of thermal spectra was performed with wide ranges in $r$, $N_{e}$ and $T_{e}$. As can be seen from the previous section, the dependence of $T_{e}$ on the spectrum when $\tau_{v} \ll 1$ is very small, hence, we have taken $T_{e}=7000 \mathrm{~K}$ for the results reported below.

Table II and Figure 1 show the main results of the computations. In Table II we compare the observed radio emission at $\lambda 3 \mathrm{~cm}$ and $\lambda 3 \mathrm{~mm}$ wavelengths (these are extrapolated fluxes; references for radio observations are given in Section 1) with the theoretically predicted pure thermal radiation. The computed flux densities due to thermal radiation in Table II are those at $\lambda 3 \mathrm{~cm}$. The thermal spectra are almost independent of wavelength when $\tau \ll 1$ and the fluxes at $\lambda 3 \mathrm{~cm}$ are essentially the same

TABLE II

Flux densities at $\lambda 3 \mathrm{~cm}, \lambda 3 \mathrm{~mm}$, extrapolated from the observed spectra, compared with predicted thermal fluxes

\begin{tabular}{|c|c|c|c|c|c|}
\hline \multirow[t]{2}{*}{ Galaxy } & \multirow[t]{2}{*}{$3 \mathrm{~cm}$ (obs.) } & \multirow[t]{2}{*}{$3 \mathrm{~mm}$ (obs.) } & \multicolumn{3}{|c|}{ Flux Density $\left(10^{-26} \mathrm{~W} \mathrm{~m}^{-2} \mathrm{~Hz}^{-1}\right)^{\mathrm{a}}$} \\
\hline & & & Thermal (1) & Thermal (2) & Thermal (3) \\
\hline M31 & 1.50 & 0.11 & 0.32 & 60.5 & 505.0 \\
\hline \multirow[t]{2}{*}{$\mathrm{M}^{3} 3^{\mathrm{b}}$} & 0.31 & 0.03 & a) 0.169 & 32.5 & 271.3 \\
\hline & & & b) 0.046 & 8.9 & 74.3 \\
\hline NGC 628 & 0.025 & 0.003 & 0.0007 & 0.135 & 1.13 \\
\hline NGC 2403 & 0.15 & 0.07 & 0.0023 & 0.448 & 3.75 \\
\hline NGC 2903 & 0.07 & 0.009 & 0.0003 & 0.063 & 0.53 \\
\hline NGC 3368 & 0.002 & 0.00002 & 0.00001 & 0.002 & 0.02 \\
\hline NGC 3627 & 0.14 & 0.03 & 0.0001 & 0.020 & 0.17 \\
\hline NGC 3628 & 0.11 & 0.02 & 0.00008 & 0.015 & 0.13 \\
\hline NGC 4449 & 0.05 & 0.004 & 0.0016 & 0.314 & 2.62 \\
\hline NGC 5457 & 0.06 & 0.007 & 0.0036 & 0.691 & 5.77 \\
\hline NGC 6946 & 0.30 & 0.05 & 0.0005 & 0.97 & 0.81 \\
\hline
\end{tabular}

a Computed thermal fluxes are at $\lambda 3 \mathrm{~cm}$. The thermal spectra are almost flat at this wavelength region so that the $\lambda 3 \mathrm{~mm}$ fluxes are essentially the same as those at $\lambda 3 \mathrm{~cm}$.

b a) 369 Hil regions, b) 101 Hil regions, (see text for observed flux).

Thermal (1) $\left\langle N_{e}\right\rangle=20 \mathrm{~cm}^{-3},\langle r\rangle=10 \mathrm{pc}$.

Thermal (2) $\left\langle N_{e}\right\rangle=70 \mathrm{~cm}^{-3},\langle r\rangle=25 \mathrm{pc}$.

Thermal (3) $\left\langle N_{e}\right\rangle=100 \mathrm{~cm}^{-3},\langle r\rangle=40 \mathrm{pc}$. 


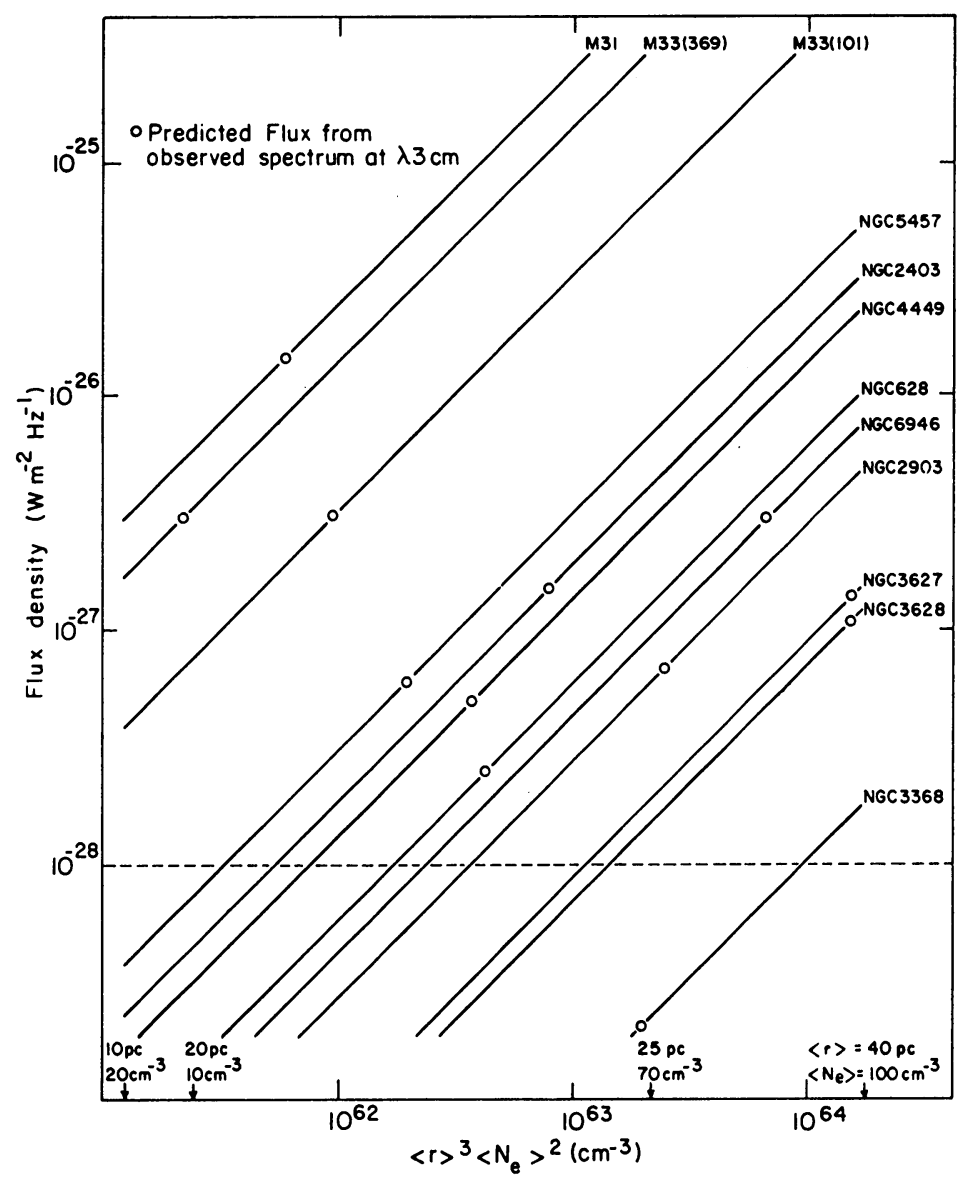

Fig. 1. Predicted flux densities due to thermal radiation for a number of galaxies as a function of $\langle r\rangle^{3}\left\langle N_{e}\right\rangle^{2} .\langle r\rangle$ is the mean radius of the HII regions and $\left\langle N_{e}\right\rangle$ is the mean electron density. The dashed line indicates the lower limit of detectability for a radio source with present-day instrumentation. The circles on each curve show the predicted flux for the corresponding galaxies at $\lambda 3 \mathrm{~cm}$ extrapolated from their observed spectra.

as those at $\lambda 3 \mathrm{~mm}$. Three cases of computed thermal spectra are given in Table II with regard to mean radii and mean densities of the HII regions; (1) $\left\langle N_{e}\right\rangle=20 \mathrm{~cm}^{-3}$, $\langle r\rangle=10 \mathrm{pc} ;(2)\left\langle N_{e}\right\rangle=70 \mathrm{~cm}^{-3},\langle r\rangle=25 \mathrm{pc} ;(3)\left\langle N_{e}\right\rangle=100 \mathrm{~cm}^{-3},\langle r\rangle=40 \mathrm{pc}$.

The data on $\left\langle N_{e}\right\rangle$ and $\langle r\rangle$ for our own galaxy can be obtained from Murdin and Sharpless (1968). These authors have compiled more than seventy emission regions and their data indicate that $\left\langle N_{e}\right\rangle \sim 70 \mathrm{~cm}^{-3}$ and $\langle r\rangle \sim 25$ pc. One can clearly note that if the mean population of $\mathrm{H}$ II regions in other spiral galaxies is similar to our own, then case (2) in Table II shows that the radio radiation of the thermal regions is in general of the same order as the radio non-thermal radiation at $\lambda \lesssim 3 \mathrm{~cm}$. One also notes that a comparison of the predicted thermal radiation with the observations may put a limit on the mean values of $r$ and $N_{e}$ (actually on the product $\langle r\rangle^{3}\left\langle N_{e}\right\rangle^{2}$ ). 
Figure 1 shows the relationship between flux density due to thermal radiation (at $\lambda 3 \mathrm{~cm}, T_{e}=7000 \mathrm{~K}$ ) and the parameter $\langle r\rangle^{3}\left\langle N_{e}\right\rangle^{2}$ for the Hil regions in eleven galaxies. The dashed line represents the low flux density limit for a detectable radio source with the present-day available instrumentation.

The arrows show four examples of combinations of $\langle r\rangle$ and $\left\langle N_{e}\right\rangle$. The open circles indicate the flux densities from the observed spectra of the galaxies (extrapolated to $\lambda 3 \mathrm{~cm}$ ). As an example, if $\langle r\rangle=20 \mathrm{pc}$ and $\left\langle N_{e}\right\rangle=10 \mathrm{~cm}^{-3}$ for M33, where Sandage reports $369 \mathrm{HII}$ regions, then we see that the flux due to the Hil regions is of the same order as the flux due to the non-thermal radiation $\sim 0.3 \mathrm{f}$.u. (Recent observations of M33 by Terzian and Pankonin (1971, in preparation) show a flux at $\lambda 3 \mathrm{~cm}$ of 4 f.u.)

One should emphasize that these computations include only the free-free radiation from normal Hil regions. No attempt has been made to include the so called 'background' thermal component, which appears in our own galaxy, and which is probably due to ionization of the neutral hydrogen by low energy cosmic rays.

As a conclusion, we have shown that thermal radiation from normal spiral galaxies may be important at centimeter wavelengths, and certainly very important at millimeter wavelengths. More accurate predictions can be made if one obtains integrated absolute fluxes of Balmer or Paschen lines of normal spiral galaxies.

\section{Acknowledgements}

The author wishes to acknowledge the computations done by $\mathrm{Mr} \mathrm{V}$. Pankonin on thermal spectra using the projected areas of HiI regions from Hodge's catalogue. This work was supported by the Arecibo Observatory. The Arecibo Observatory is operated by Cornell University under contract to the National Science Foundation and with partial support from the Advanced Research Projects Agency.

\section{References}

Baade, W. and Arp, H.: 1964, Astrophys. J. 139, 1027.

Bergh, van den, S.: 1968, Commun. David Dunlap Obs. 195, 1.

Burgess, A.: 1958, Monthly Notices Roy. Astron. Soc. 118, 477.

Carranza, G., Courtès, G., Georgelin, Y., Monnet, G., and Pourcelot, A.: 1968, Ann. Astrophys. 31, 63.

Courtès, G. and Cruvellier, P.: 1965, Ann. Astrophys. 28, 683.

De Jong, M. L.: 1966, Astrophys. J. 144, 553.

De Jong, M. L.: 1967, Astrophys. J. 150, 1.

Heeschen, D. S. and Wade, C. M.: 1964, Astron. J. 69, 277.

Hodge, P. W.: 1969, An Atlas and Catalog of $H_{n 1}$ Regions in Galaxies, University of Washington, Washington, D.C.

Howard, W. E., III and Maran, S. P.: 1965, Astrophys. J. Suppl. Ser. 10, 1.

Kraus, J. D., Dixon, R. S., and Fisher, R. O.: 1966, Astrophys. J. 144, 559.

Kuril'chik, V. N., Andrievskii, A. E., Ivanov, V. N., and Spangenberg, E. E.: 1970, Soviet Astron. 13, 881.

Lang, K. R. and Terzian, Y.: 1969, Astrophys. Letters 3, 29.

Mathewson, D. S. and Rome, J. M.: 1963, Australian J. Phys. 16, 360. 
Murdin, P. and Sharpless, S.: 1968, in Y. Terzian (ed.), Interstellar Ionized Hydrogen, W. A. Benjamin Press, New York, p. 289.

Oster, L.: 1961, Astron. J. 134, 1010.

Pipher, J. L. and Terzian, Y.: 1969, Astrophys. J. 155, 475.

Roberts, M. S.: 1969, Astron. J. 74, 859.

Sandage, A. R.: 1962, in G. C. McVittie (ed.), 'Problems of Extra-Galactic Research', IAU Symp. 15, 359.

Terzian, Y.: 1965, Astrophys. J. 142, 135.

Terzian, Y.: 1968, in D. E. Osterbrock, and C. R. O'Dell (eds.) 'Planetary Nebulae', IAU Symp. 34, 87. Whiteoak, J. B.: 1970, Astrophys. Letters 5, 29. 